\title{
Polyethylene glycol (PEG)-based peptidomimetics (Pegtides)
}

\author{
Marc Devocelle, Alan Hibbitts, Aoife O’Connor, Sally-Ann Cryan, Deirdre Fitzgerald-Hughes
}

RCSI, Ireland

https://doi.org/10.17952/35EPS.2018.175

Peptide and protein drug discovery generates potent leads in most therapeutic areas.[1] Their optimisation and development may involve peptidomimetic conversion, or conjugation to biocompatible polymers. The features of these two techniques are combined in polymer-based peptidomimetics. For instance, functionalised poly(norbornene), poly(acrylic) and nylon-3 backbones have been described as synthetic mimics of Antimicrobial Peptides (AMPs).[2, 3] Polyethylene Glycol (PEG), one of the most established biocompatible polymers for improving the pharmacokinetic and pharmacodynamic properties of polyamide-based therapeutics, has not been exploited as a backbone for peptidomimetics on the other hand.[4]

Co- and post-polymerisation functionalisation of substituted ethylene oxides can produce PEG-based backbones with branching chains separated by an equal number of bonds than side chains in peptides (Fig. 1). These hybrid structures of PEG and peptides, termed 'Pegtides', are different from PEGtide dendrons which alternate monodisperse non-functionalised PEG chains and dipeptide motifs.[5]

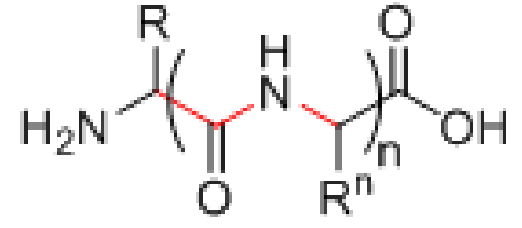

Peptide

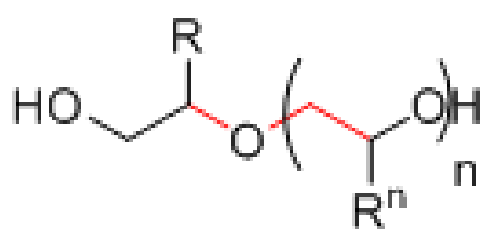

Pegtide

Figure 1: General structures of peptides and PEG-based peptidomimetics 'pegtides'.

Homopolymerisation and copolymerisation of substituted oxiranes, followed by possible functional group interconversion, can produce PEG-based peptidomimetics of poly ( $\alpha$-amino acids) and of sequences enriched in two types of amino acids, respectively. Hence, copolymers with hydrophobic and positively charged repeating units can mimic amphipathic peptides such as AMPs. Their sequences are indeed characterised by a net excess of positively charged residues and a hydrophobic amino acid content approximating 30-50\%.[6] Moreover, the main activity determinants of these peptides are their overall amino acid content and sequence pattern, rather than sequence-dependent arrangement. These characteristic features can be mimicked with statistical copolymers. Consequently, an AMP pegtide was prepared by anionic ring-opening copolymerisation of tert-butyl N-(2-oxiranylmethyl)carbamate and 1,2-epoxyhexane, using sodium benzyloxide as the initiator, followed by Boc removal by acidolytic cleavage and guanylation of the resulting amines with N,N'-diBoc-1H-pyrazole-1-carboxamidine. Final deprotection by treatment with trifluoroacetic acid yielded poly(hexene oxide-co-glycidylguanidine)s (Fig. 2, left), which were purified by Size-Exclusion Chromatography (SEC) or precipitation and analysed by ${ }^{1} \mathrm{H}$ NMR and MALDI-TOF Mass Spectrometry. Short copolymers isolated by SEC $(m=1-2, n=2-3)$ were tested in preliminary susceptibility and antibiofilm assays. The former indicated Minimum Inhibitory Concentrations (MICs) of 150-300 $\neq \mathrm{M}$ against the model Gram-positive ( Staphylococcus aureus ) and Gram-negative (Escherichia coli) organisms. These pegtides also showed some growth inhibitory properties in a Staphylococcus epidermidis biofilm prevention a ssay (Fig. 3). Longer copolymers with 4 glycidylguanidine $(\mathrm{m}=4)$ and 10 hexene oxide repeating units $(\mathrm{n}=10)$ were also obtained by this approach. 

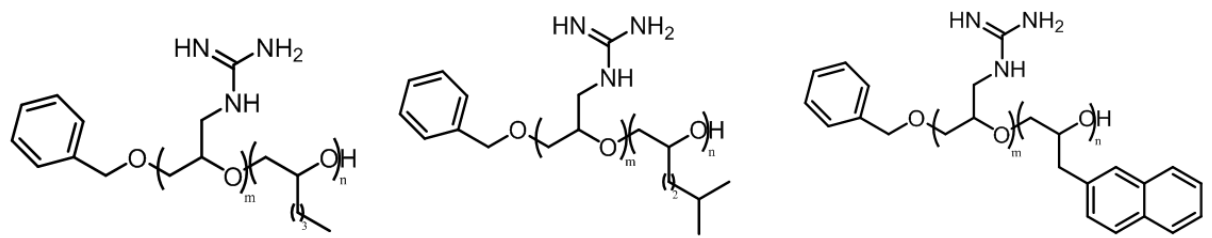

Figure 2: Pegtides of (arginine, norleucine)-, (arginine, homoleucine)- and (arginine, tryptophan)-rich AMPs.
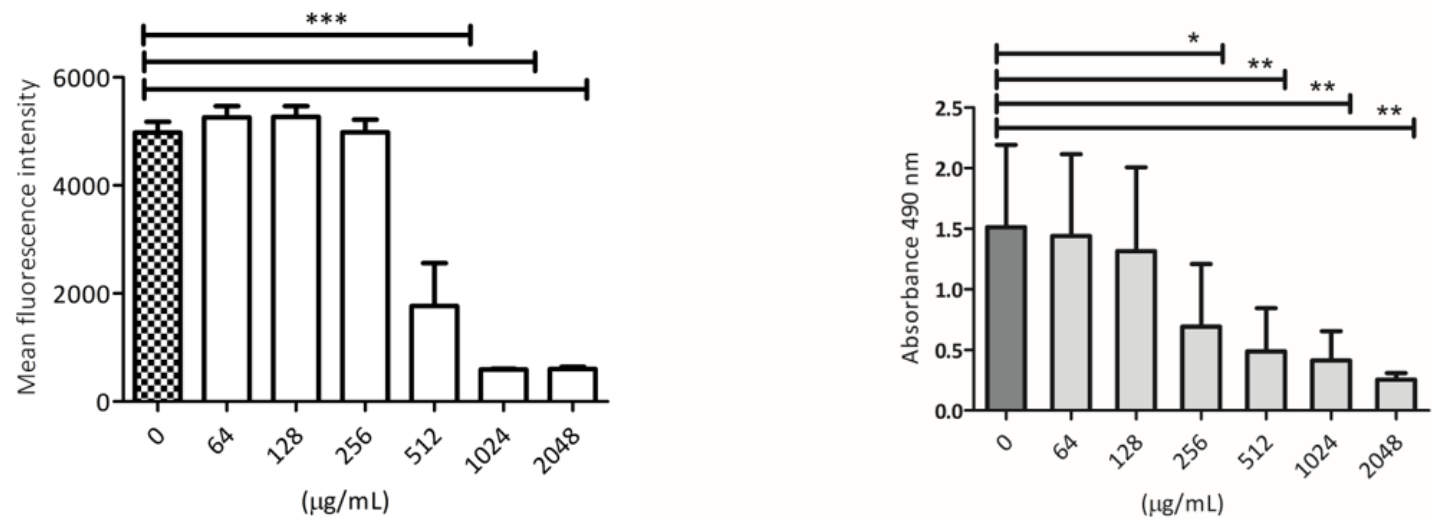

Figure 3: Prevention of S. epidermidis biofilm formation by short Arg-Nle mimetics; (left) viability of cultures determined by a Resazurin-conversion assay; (right) biofilm formation quantified by the crystal violet staining method. Presented are means $\pm S D$ of the independent experiments. Statistical significance was determined by 2-way ANOVA with Dunnett's multiple comparison test, $* P<0.05 * * P<0.005 \& * * * P<0.001$.

Two other AMP pegtides, differing by their hydrophobic contents, were prepared by using epoxide monomers mimicking branched or aromatic amino acids. They were 2-(3-methylbutyl)oxirane for homoleucine (Fig. 2, centre) and 2-(naphtalen-2-ylmethyl)oxirane for 3-(2-naphtyl)alanine, itself a substitute of tryptophan (Fig. 2, right). Copolymers were obtained with degrees of polymerization of 10 on average. Preliminary susceptibility testing indicated that peptidomimetics based on branched lipophilic units displayed greater antimicrobial activities than their linear counterparts and that the Arg-Trp pegtides displayed the lowest MICS, one dilution away from those of their parent decapeptide, against both Gram-negative and Grampositive bacteria.

\section{Acknowledgements}

This material is based upon works supported by the Science FoundationIreland underGrant No. 13/TIDA/ B2657. The authors would like to thank Dr Andrea Molero Bondia and Mr Daniel O'Neill for the synthesis of the peptidomimetics, Drs Deirdre Fitzgerald-Hughes, Eoghan O'Neill and Marta Zapotoczna for the susceptibility testing.

\section{References}

1 Henninot A, Collins JC, Nuss JM. The CurrentState of Peptide Drug Discovery: Back to the Future? J Med Chem. 2018, 61(4):1382-1414.

2 Lienkamp K, Tew GN. Synthetic mimics of antimicrobial peptides-a versatile ring-opening metathesis polymerization based platform for the synthesis of selective antibacterial and cell-penetrating polymers. Chemistry. 2009, 15(44):11784-11800.

3 Dorner F, Lienkamp K, in Polymeric Materials with Antimicrobial Activity: From Synthesis to Applications, ed. A. Munoz-Bonilla, M. Cerrada, M. Fernández-García, Royal Society of Chemistry, Cambridge, UK, 1st edition, 2013, chapter 5, 97-138.

4 Herzberger J, Niederer K, Pohlit H, Seiwert J, Worm M, Wurm FR, Frey H. Polymerization of Ethylene Oxide, 
Propylene Oxide, and Other Alkylene Oxides: Synthesis, Novel Polymer Architectures, and Bioconjugation. Chem Rev. 2016, 116(4):2170-2243.

5 Gao J, Chen P, Singh Y, Zhang X, Szek ely Z, Stein S, Sinko PJ. Novel monodisperse PEGtide dendrons: design, fabrication, and evaluation of mannose receptor-mediated macrophage targeting. Bioconjug Chem. 2013, 24(8):1332-1344.

6 Zasloff M. Antimicrobial peptides of multicellular organisms. Nature. 2002, 415(6870):389-395. 